\title{
Aerodynamic Testing and Development of Sunswift eVe
}

\author{
Simon Ambrose* \\ UNSW Australia, Sydney, NSW 2052, Australia \\ Graham Doig ${ }^{\dagger}$ \\ California Polytechnic State University, CA 94307 \\ UNSW Australia, Sydney, NSW 2052, Australia
}

\begin{abstract}
The current understanding of the aerodynamic performance of Sunswift's solar-electric race car eVe is limited, despite the design and manufacture of the vehicle in 2012-13. This paper describes an investigation into the aerodynamic behaviour of the vehicle and details the successive design and development of drag minimisation strategies. A study of the external airflow around the vehicle was undertaken through a computational fluid dynamics analysis, with validation offered through the results of real-world track testing. Particular reference is made to the Sunswift team's successful long-range electric vehicle land speed record attempt on 23 July 2014. A predicted $10 \%$ reduction in drag has been achieved through external bodywork modifications as described within this paper. Recommendations for the design of low drag vehicles are also presented, with relation made to the future of sustainable transport.
\end{abstract}

\section{Nomenclature}

$\rho \quad$ Fluid density, $\mathrm{kg} / \mathrm{m}^{3}$

A Projected frontal area, $\mathrm{m}^{2}$

$C_{D} \quad$ Coefficient of Drag

$C_{D} A$ Drag area, $\mathrm{m}^{2}$

$C_{L} \quad$ Coefficient of Lift

$C_{r r} \quad$ Coefficient of rolling resistance

$g \quad$ Acceleration due to gravity, $\mathrm{m} / \mathrm{s}^{2}$

$m \quad$ Vehicle mass, $\mathrm{kg}$

P Power, W

$v \quad$ Vehicle velocity, $\mathrm{m} / \mathrm{s}$

CFD Computational Fluid Dynamics

Li-ion Lithium ion

RANS Reynolds Averaged Navier-Stokes

SST Shear Stress Transport

WSC World Solar Challenge

\footnotetext{
*Undergraduate Student, UNSW Australia, School of Mechanical and Manufacturing Engineering, Kensington AIAA Student Member

${ }^{\dagger}$ Assistant Professor, Aerospace Engineering Department, San Luis Obispo Co-appointment: Adjunct Lecturer, UNSW Australia, School of Mechanical and Manufacturing Engineering Kensington, AIAA Senior Member
} 


\section{Introduction}

erodynamic performance has long been an important consideration in the design of automobiles. At A the simplest level, the ability for a vehicle to travel through the surrounding air with minimal resistance offers numerous performance benefits in terms of maximum speed, acceleration and energy efficiency. Currently in an era where concerns over the hazardous nature and depletion of conventional fuel resources are on the rise, the future of the automotive industry appears to be geared towards one that values sustainable and energy efficient design. Many commercial alternative energy vehicles have already begun to display future promise.

However, the present resistance towards the wide-spread adoption of sustainable transport is society's apprehension in accepting a compromise in vehicle performance. Compared against conventional internal combustion vehicles, current alternative energy transport technologies struggle to match key performance characteristics such as power, range and maximum speed. As aerodynamic forces represent the dominant force opposing the forward motion of a vehicle at high speed, design for low drag presents the potential for significant performance gains. Particularly in the case of solar-powered vehicles, whose energy resources are substantially less than their fossil fuel counterparts, the necessity of an optimised aerodynamic design is crucial in closing the gap between these two propulsion technologies.

Investigations into low drag vehicle shapes have been conducted since the 1920's, with Klemperer's early design of a basic body with wheels achieving a $C_{D}=0.15$. While drag values for passenger cars have been steadily decreasing, mass manufactured vehicles have yet to achieve the low drag coefficients shown by such early research. ${ }^{8}$ Early vehicle performance was mainly restricted by available engine power and road quality, and hence didn't necessitate the need for an efficient aerodynamic design. Similarly, a study by Le Good revealed that exterior vehicle style is amongst the top three sales attributes in any market segment, if not the top attribute itself..$^{5}$ Minimising the aerodynamic drag of a vehicle, is by no means a straight-forward task. Numerous challenges arise due to the necessity of incorporating many functional design elements such as wheels, suspension, engine, transmission, wing mirrors, passenger seating and storage space into an aesthetic aerodynamic package. This challenge is made all the more difficult due to the inherently complex airflow over a vehicle body in proximity to the ground. It is highly three dimensional, and doesn't necessarily follow the contours of entire vehicle surface. ${ }^{4}$ The presence of turbulent flow structures are an inherent feature to automotive flows, typically characterised by unsteady flow around the wheels and a trailing low pressure wake behind the vehicle. Separated flow regimes are common, and are a primary source of aerodynamic drag. ${ }^{9}$

There are two components to aerodynamic drag; pressure drag and viscous drag. Pressure drag arises due to the uneven distribution of pressure around a vehicle. This is the dominating drag component for bluff body forms that are typically adopted by conventional passenger vehicles. Viscous drag or skin friction drag arises due to the frictional shearing of fluid molecules tangential to the vehicle surface. This is the dominating drag force component for streamlined body forms such as solar vehicles, where flow separation effects are minimal. Sunswift eVe is essentially a hybrid of these two forms, incorporating the practical elements of passenger vehicles while maintaining a streamlined design (Fig. 1).

\section{A. Background}

The UNSW Solar Racing Team "Sunswift" is a team of students at UNSW who compete in the World Solar Challenge, a biennial race event aimed at encouraging research and development into solar-powered vehicles. The event traverses just over $3,000 \mathrm{~km}$ of the Australian outback in a race from Darwin to Adelaide. The introduction of the Cruiser competitive class as part of the 2013 WSC placed a focus on the design of a practical solar vehicle, rewarding teams for incorporating functional design elements such as passenger seating and luggage space. After achieving previous success in the Challenger class with Sunswift IVy, the team shifted their focus towards the design of a more practical solar vehicle. Over the course of 18 months and in accordance with design criteria outlined by the WSC committee, the team undertook the design and construction of their fifth solar vehicle "eVe" in time for the 2013 WSC (Fig. 1).

Deviating from traditional streamlined solar vehicle designs, eVe takes the form of a hybrid solar-electric car resembling more of a modern sports car shape, featuring four wheels and two seats. Sunswift eVe features a 
$4 \mathrm{~m}^{2}$ silicon solar array generating a total power output of $850 \mathrm{~W}$. Coupled with Li-ion rechargeable batteries providing a storage capacity of $16 \mathrm{kWh}$, eVe has a maximum range of $800 \mathrm{~km}$ in sunny conditions and a theoretical top speed of $140 \mathrm{~km} / \mathrm{h}$.

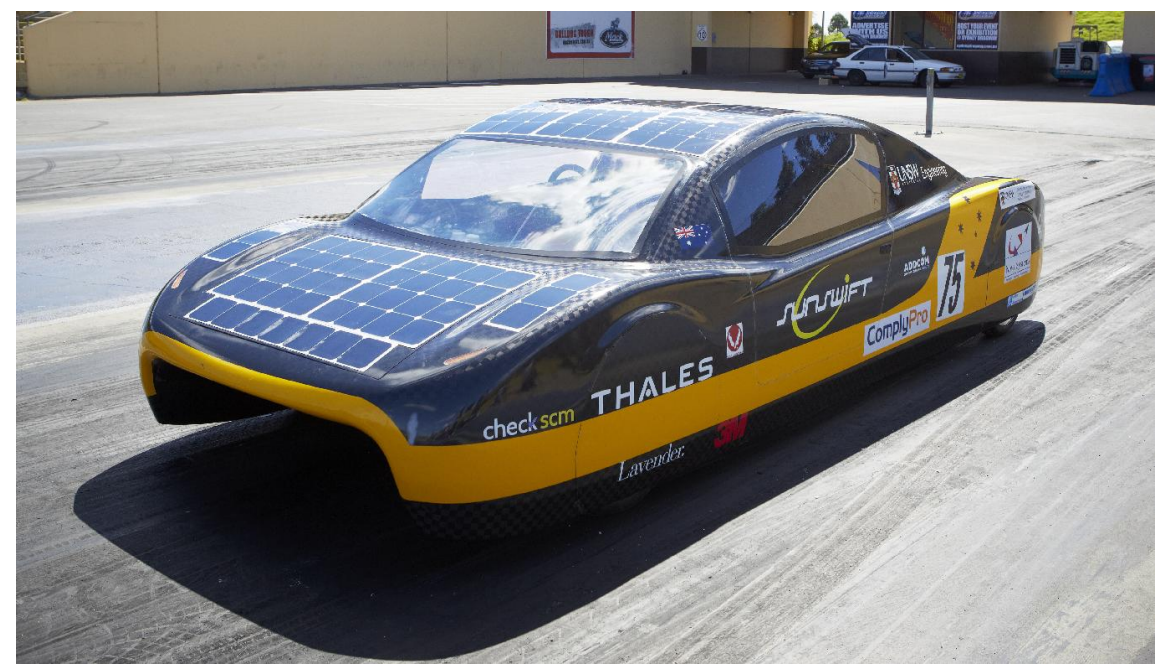

Figure 1. Sunswift eVe. Image courtesy Nikki To

\section{B. Scope of this Research}

While aerodynamic performance was highly considered during the external shape development of eVe, the need for the vehicle to be completed in time for the 2013 WSC meant that various aerodynamic optimisations to the external body surface could not be attempted. The relatively rapid shape development phase of the vehicle was conducted in the context of optimising lift and drag values, and a complete aerodynamic assessment of the vehicle was not undertaken.

Going into 2014, the Sunswift team had set themselves the goal of breaking the land speed record for the fastest long-range electric vehicle. This presented an incentive to pursue an investigation into the aerodynamic behaviour of the vehicle, with the primary outcomes of identifying sources of drag due to the current vehicle design, leading into the subsequent development of drag minimisation strategies. As the car was primarily designed to race in accordance with various design constraints imposed by the WSC committee, such limitations would not be in effect during the record attempt. This presented an opportunity to modify the external geometry of the vehicle to exploit reduced drag potential. As it is not within the team's budget to undertake a complete redesign of the vehicle, this study instead focused on the development of modular drag-reducing bodywork attachments to assist the Sunswift team in their land speed record attempt.

\section{Methodology}

$\mathrm{I}^{\mathrm{N}}$

NVESTIGATIONs into the aerodynamic behaviour of Sunswift eVe were conducted using CFD. To establish a basis for the design and development of drag reducing bodywork, an initial aerodynamic analysis was undertaken of the existing setup of Sunswift eVe. The objectives of this preliminary analysis were to visualise the flow field in the vicinity of the vehicle and identify sources of drag due to external geometry. This initial assessment would also provide a basis with which to evaluate the effectiveness of subsequent drag reducing designs against.

As the outcomes of this study are largely dependant on the results obtained through computational simulations, it was necessary to ensure the validity and accuracy of the computational model in representing a realistic flow environment. A verification study was undertaken investigating variations in grid density and boundary size to ensure grid-independent solutions. Similarly, validation of the computational results was undertaken through real world track testing of Sunswift eVe. 


\section{A. Design of the Computational Model}

The CATIA CAD package was used to prepare and modify the vehicle geometry used within this study. To facilitate the mesh generation process, the wheels were set onto small steps of $1.5 \mathrm{~mm}$ height to minimise the formation of highly skewed elements as a result of the near-tangent contact of the wheel on the ground. ${ }^{2}$ Similarly, due to the inherent complexities in doing so, the front and rear wheel wells of the vehicle were not modelled. Instead, this region of the vehicle was modelled as a smooth continuous surface with the wheels protruding outward towards the ground. To assist in the aerodynamic analysis, the CAD geometry representing Sunswift eVe was sectioned into geometric surface feature groups. These surface groups were incorporated into the solution process and allowed for the resultant pressure and viscous force components acting on each surface to be reported.

\section{Mesh Design}

The Cutcell assembly meshing algorithm with ANSYS Meshing Workbench was used to mesh the fluid domain, having the benefit of producing a large fraction of hexahedral elements with faces that are aligned with the coordinate axes (and hence the main flow direction). ${ }^{1}$ A denser mesh was set up in proximity of the vehicle in order to better resolve the stronger flow gradients expected in these regions. The majority of the vehicle surface was resolved with an $8 \mathrm{~mm}$ cell size, with further refinement implemented on the wheels, underskirt and leading edges of the vehicle (Fig. 2). Smaller geometry details such as surface fillets were captured using a $2 \mathrm{~mm}$ cell sizing. The region directly behind Sunswift eVe was modelled with a finer resolution mesh to better resolve the wake produced by the vehicle.

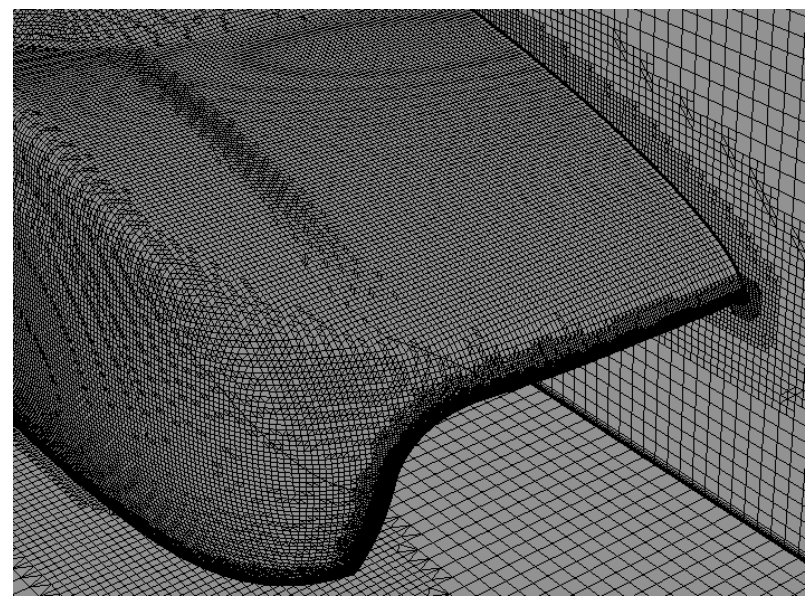

(a) Surface mesh

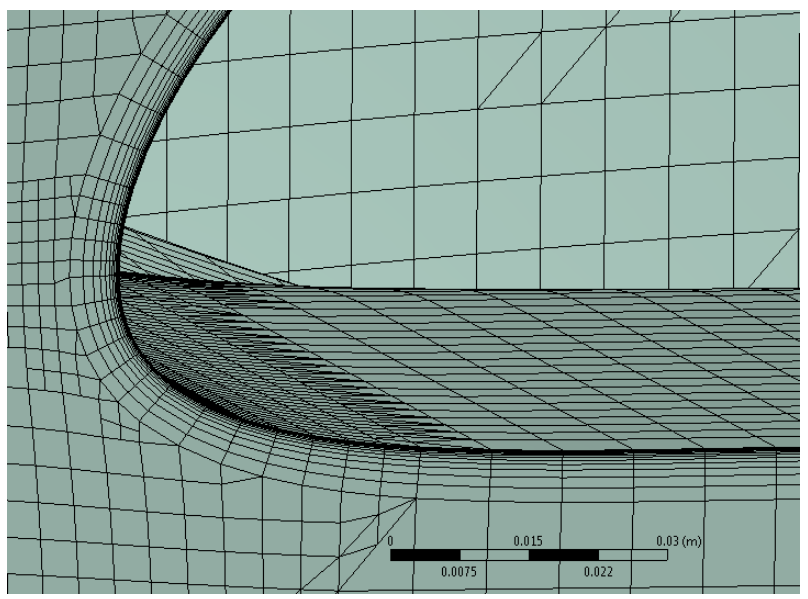

(b) Boundary layer mesh

Figure 2. Mesh design around the leading edge of Sunswift eVe

A near-wall modelling approach was adopted to accurately resolve the boundary layer formation around the vehicle and obtain a better estimate of drag due to skin friction. A structured mesh composed of 14 layers was inflated from the surface of the vehicle. The growth rate and first cell height of this inflation layer were configured to achieve an average $y^{+} \approx 1.5$ on the vehicle surface while minimising the percentage volume change between the last inflation layer cell and neighbouring CutCell element. A similar inflation layer was implemented on the ground surface and configured to ensure $y^{+} \approx 1$ in the proximity of the vehicle.

\section{Boundary Conditions}

The boundary of the fluid domain was set to $60 \mathrm{~m}$ length, $30 \mathrm{~m}$ width and $18 \mathrm{~m}$ height, corresponding to a model blockage ratio of $0.2 \%$. This was to ensure minimal interference effects due to the proximity of the walls. The centre of the vehicle was placed $20 \mathrm{~m}$ from the inlet, leaving a $40 \mathrm{~m}$ region for the wake to develop as displayed in Fig. 3. For straight line simulations, a symmetry boundary condition was applied down the centreline of the vehicle effectively halving the width of the domain in order to reduce the computational and time cost of the simulations. Runs were conducted at $35 \mathrm{~m} / \mathrm{s}$ inlet velocity, to model the upper range of speeds that Sunswift eVe is able to achieve. The outlet face was set as a zero pressure outlet. The ground 


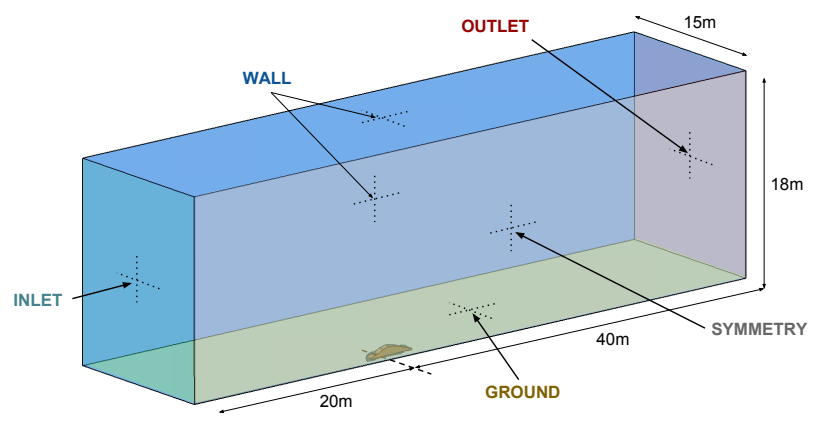

Figure 3. Domain setup used in CFD analysis

face of the fluid domain was set as a non-slip translating boundary, with translational velocity equal to the inlet velocity to simulate a moving road surface. The far walls of the fluid domain were modelled as zero shear walls to prevent the unnecessary calculation of a boundary layer on these faces. Similarly, the small steps that the wheels are situated on are also modelled using the zero shear wall condition to better simulate a tyre in contact with the ground. The wheels of the vehicle are modelled as a rotating non-slip boundary, with a rotational velocity specified at the axis of the wheel, consistent with the inlet and ground velocities.

\section{Solution Setup}

FLUENT 14.5's steady-state RANS solver was utilised in this study. Solutions were obtained using secondorder spatial discretisation for the flow variables using the SIMPLE solver, with cases run until residual values had converged to a minimum level of $10^{-4}$. The presence of rotating wheels within the model caused instabilities in monitored lift and drag coefficients. These values were determined by averaging across the last 1,000 solution iterations, ensuring that lift and drag values had reached a point of stable oscillations about an apparent mean value. This equated to 6,000 iterations in most cases. The Transition SST turbulence model was chosen for this study, given its apparent success in the design and development of the Umicar III solar vehicle, ${ }^{3}$ recommended for its accuracy in predicted flow separation points, laminar to turbulent boundary layer transition and strong similarity to wind tunnel test results.

\section{Verification}

$\mathrm{F}^{\mathrm{n}}$ IVE meshes of varying grid density were investigated to assess the solution dependency on mesh grid resolution. The total number of elements between the five grid sizes were varied by proportionately adjusting global and local mesh sizing controls. The results in Fig. 4a are shown relative to the largest grid size of 16.3 million elements. Similarly, investigations into boundary size in Fig. 4b considered four different domain sizes, scaled relative to the boundary dimensions shown in Fig. 3.

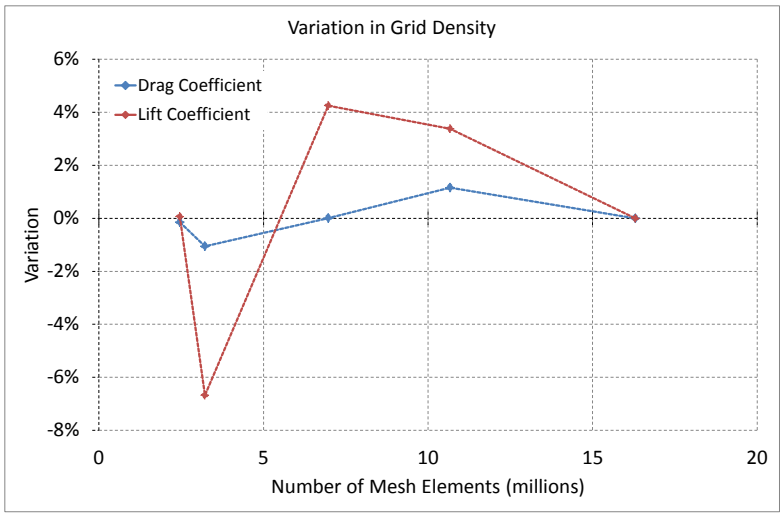

(a) Grid convergence study

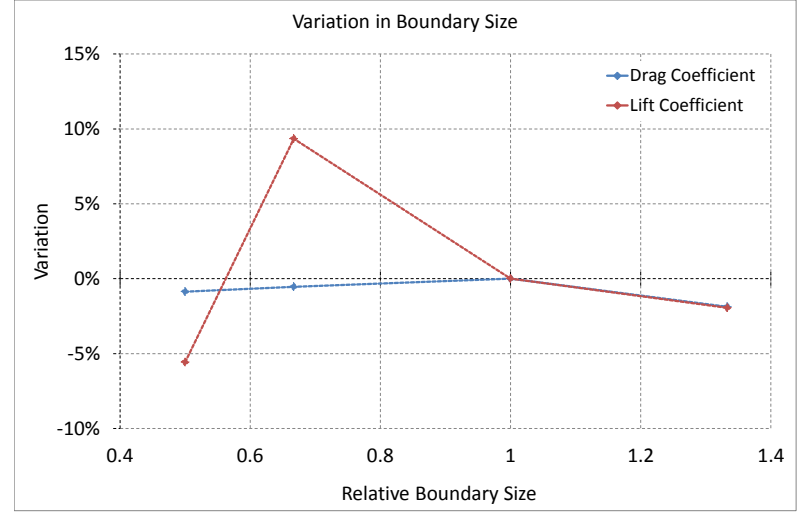

(b) Boundary size investigation

Figure 4. Verification study investigating grid resolution and boundary size 
In both studies, predicted drag coefficients showed a good degree of consistency, however predicted lift coefficients showed greater variation. Across all cases investigated, the monitored lift coefficient displayed a greater variance in comparison to the drag coefficient during the solution process. It was discovered that this was due to unsteady flow around the wheels affecting the resultant pressure force acting on the underside surface of eVe. However, as drag values were more important to this study, the consistency between drag predictions indicated grid-independence, and a 12 million element mesh for a half-car model, along with the domain size shown in Fig. 3, was selected as the computational model basis used in this study.

\section{Current eVe Analysis}

$\mathrm{T}$ HE surface force reports indicated that pressure and viscous drag components formed a relatively even contribution to the total aerodynamic drag of the vehicle. This was further indicative that Sunswift eVe is a hybrid between bluff and streamlined body forms. The bonnet of the vehicle was shown to have the largest contribution to the total drag due to the formation of a high pressure stagnation region at the leading edge. Similarly, the majority of the lift force arose from the accelerated airflow within the underside tunnel region of the vehicle. The reduced cross-sectional area in this region results in a lower pressure which effectively sucks the vehicle to the ground.

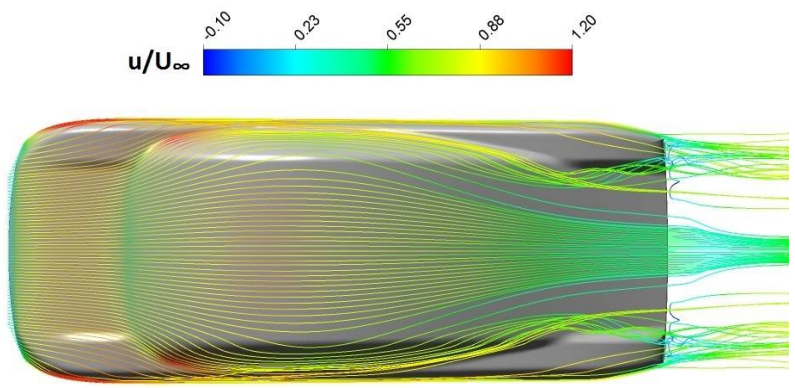

(a) Top

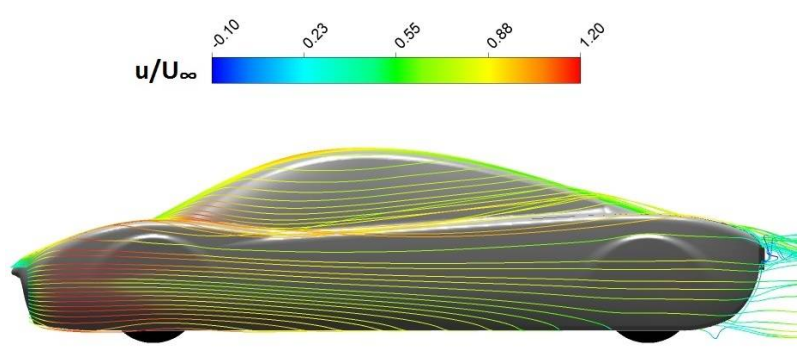

(b) Side

Figure 5. Streamlines highlighting the airflow around Sunswift eVe

The streamline plots in Fig. 5 offer some information regarding the quality of the flow over the vehicle surface. We can see that the flow remains largely attached on the front-most part of Sunswift eVe. However, towards the rear, air spills over the rear wheel shoulder as it is drawn in towards the centre of the vehicle. There is also evidence of a strong underbody suction along the side skirt between the front and rear wheels. This has the undesired effect of producing a yawed flow ahead of the rear wheel (Fig. 6). As a result, the rear wheel generates a considerably large wake directed towards the centreline of the vehicle, which then develops into a trailing vortex.

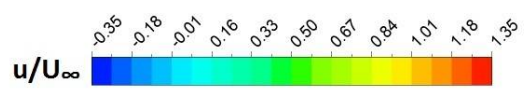

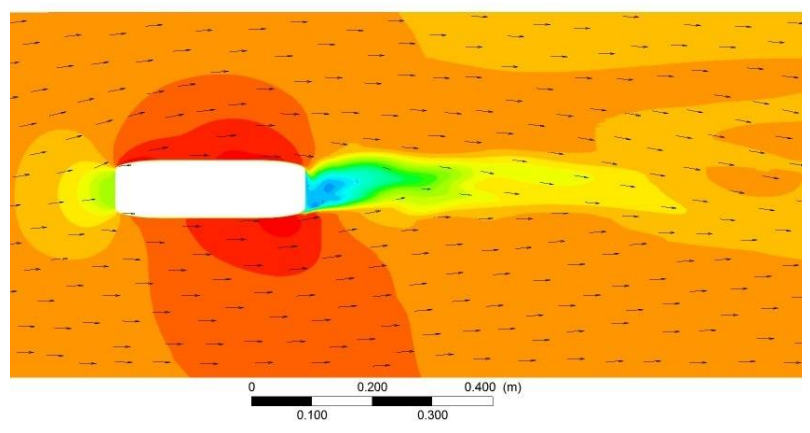

(a) Front right wheel

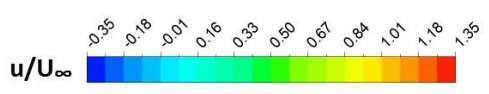

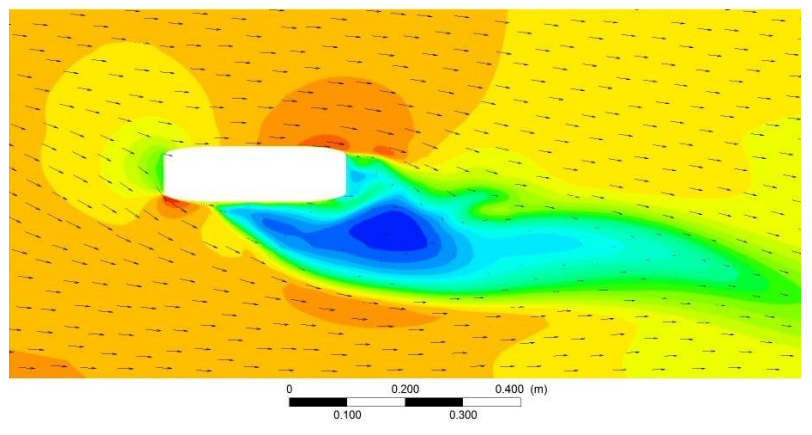

(b) Rear right wheel

Figure 6. Normalised x-velocity around the wheels at $50 \mathrm{~mm}$ from the ground 


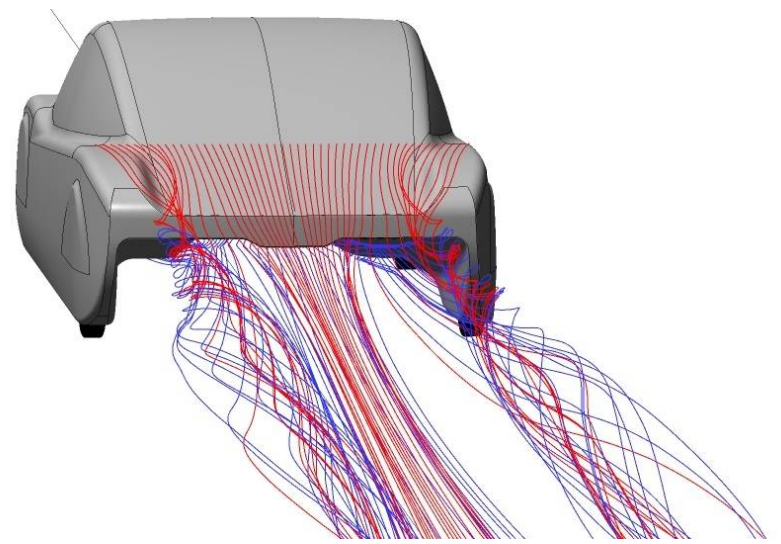

Figure 7. Flow off the rear surface of eVe. Top surface flow (red), underside surface flow (blue)

A dominating flow feature is the structure of the wake that Sunswift eVe produces, due to the interaction of airflow off the top and underside surfaces. At the rear of the top surface, air is sucked inward towards the centreline of the vehicle. In doing so, airflow is pulled over the top of the rear wheel shoulder, producing turbulent flow. Conversely, on the underside of the vehicle, the presence of the lowered license plate surface within the tunnel region has the effect of pushing the airflow out towards the side. The combination of these two flow structures at the rear is magnified by the low pressure wake immediately behind the vehicle, and results in the production of two large contra-rotating vortices which can be seen in Fig. 7.

The contra-rotating vortices also have the effect of producing a strong downwash off the rear of the vehicle. This is discussed in further detail in Fig. 11 on page 9.

\section{Validation}

$\Lambda \mathrm{N}$ attempt to validate the drag coefficient of eVe determined computationally was undertaken through A real-world constant speed testing of the vehicle. An experimental procedure for doing was adopted from A. Boulgakov's 2011 thesis, describing the race strategy and modelling of Sunswift IVy ahead of the 2011 WSC. ${ }^{6}$ Assuming testing is conducted over a flat terrain, power losses due to road gradients can be neglected and the total power consumption model of the vehicle is then dependent on losses due to rolling resistance and aerodynamic drag as expressed in Eq. 1.

$$
P_{\text {total }}=P_{\text {rolling resistance }}+P_{\text {aerodynamic drag }}=C_{r r} m g v+\frac{1}{2} C_{D} A \rho v^{3}
$$

Constant speed testing involves accelerating the vehicle to a set speed, and then cruising at that speed for a length of time. The average power output to maintain that speed is recorded, and the test is repeated for a range of set speeds to build a relationship between vehicle power as a function of velocity as theoretically expressed in Eq. 1. The result of this testing is displayed in Fig. 8.

From the resulting regression curve fit to the test data, the drag area was determined experimentally to be $C_{D} A=0.126 \mathrm{~m}^{2}$. In comparison, the computationally predicted drag area was $C_{D} A=0.146 \mathrm{~m}^{2}$. The lower drag estimate obtained experimentally is likely an unrealistic value. Unfortunately the weather conditions during the testing session were far from ideal. There was a significant presence of atmospheric wind, which varied in strength and direction throughout the test day. This would have the effect of increasing or decreasing the vehicle power draw depending on the direction of travel. In the aerodynamic development of the Tesla Model S, Tesla experienced similar difficulties in achieving reliable data through road testing. ${ }^{7}$ It was only after discounting runs with windspeeds greater than $2.5 \mathrm{~m} / \mathrm{s}$ that Tesla could achieve a degree of correlation between experimental and theoretical values. Unfortunately for this study, further testing runs in low wind conditions are required before a reliable estimation of the drag of eVe can be made. 


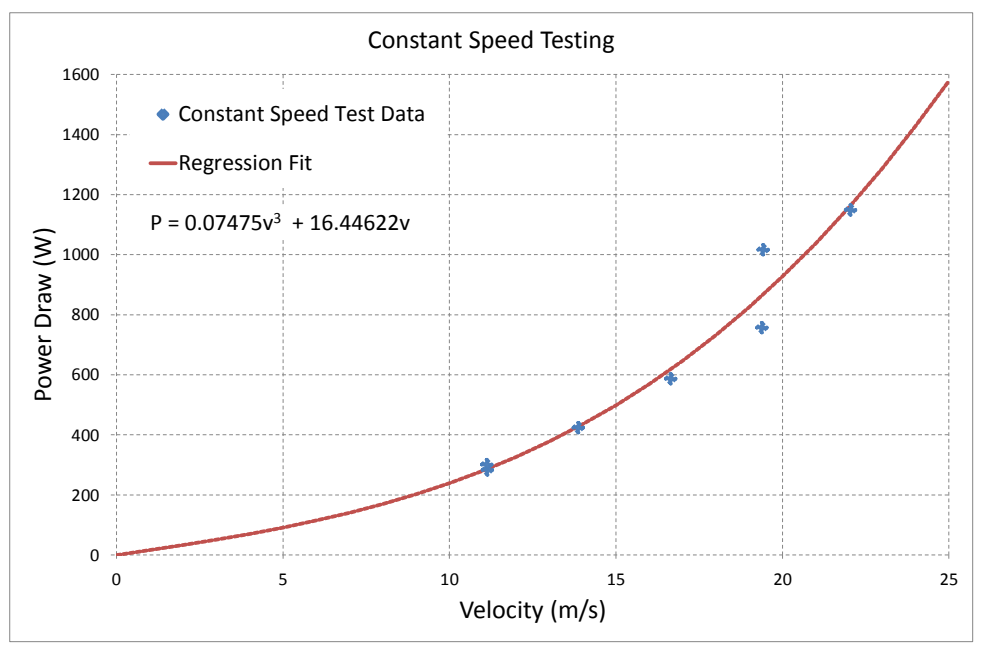

Figure 8. Constant speed testing results

\section{Design of a Trailing Edge Extension}

A significant source of drag for Sunswift eVe was recognised due to the behaviour of airflow off the rear surfaces interacting with the low pressure wake directly behind vehicle. A relatively straightforward solution to alleviate this behaviour was to adopt a low drag design approach known as "boat-tailing". ${ }^{4}$ This design approach minimises flow separation off the rear edges by extending the rear surfaces to "fill in" the region of low pressure immediately behind the vehicle.

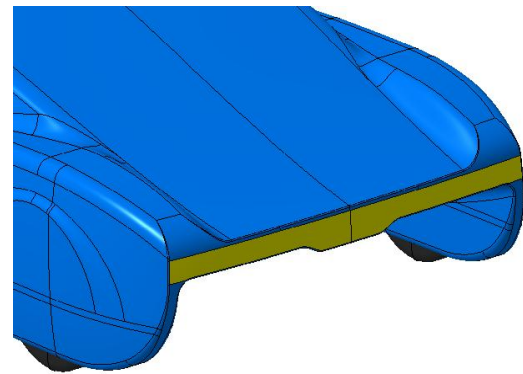

(a) Current eVe

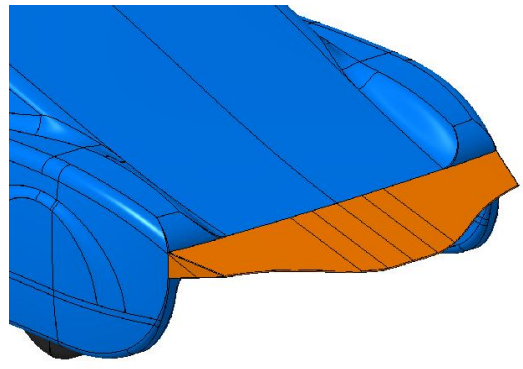

(b) Initial trailing edge extension

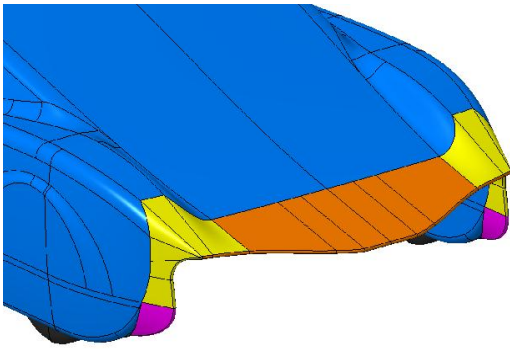

(c) Final trailing edge extension

Figure 9. Design stages of the trailing edge extension

Development of the trailing edge extension was accomplished in two phases. An initial design focused on the extension of the larger horizontal rear surface as highlighted in Fig. 9a. The upper and lower surface profiles were naturally extended until they formed an intersection $0.45 \mathrm{~m}$ behind the rear face of the vehicle. A CFD evaluation of the initial trailing edge design shown in Fig. 9b predicted a drag reduction of $3.4 \%$ compared with the original car.

Following this successful drag reduction, the trailing edge extension was improved to account for the top of the rear wheel shoulder, as well as the vertical rear faces of the vehicle (Fig. 9c). CFD analysis of the final trailing edge extension design indicated a greater drag reduction of $10.3 \%$ in comparison with the original design of Sunswift eVe.

To better understand the structure of the turbulent wake produced by the vehicle with and without the final trailing edge extension design, plots of the total pressure coefficient were extracted at several transverse planes located behind the vehicle as shown in Fig. 10. Figure 11 displays the wake produced by the current car in comparison with the wake generated by the vehicle with the final trailing edge extension attached (Fig. 12). 


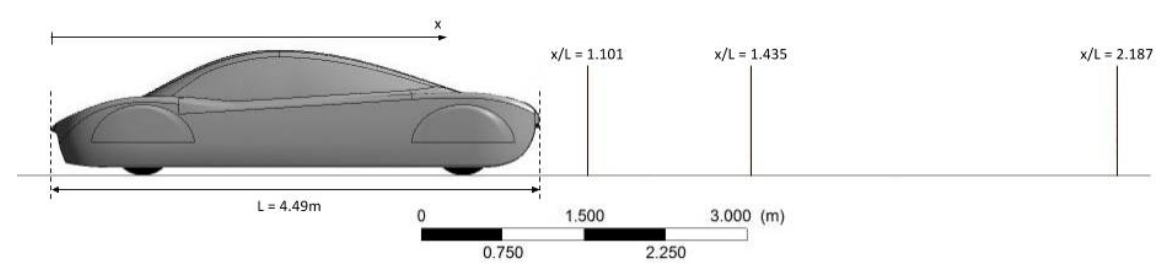

Figure 10. Locations of transverse planes used for wake analysis

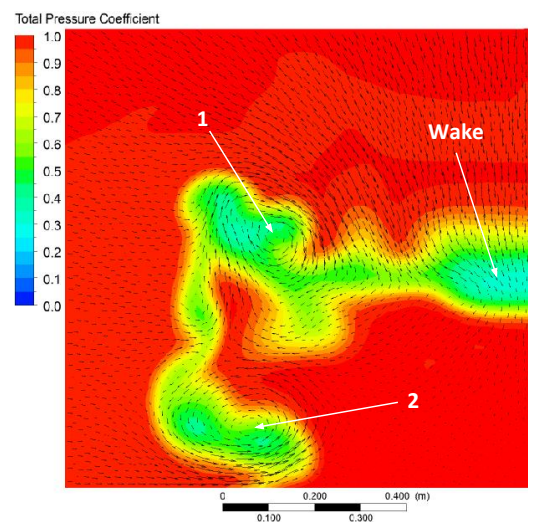

(a) $\mathrm{x} / \mathrm{L}=1.101$

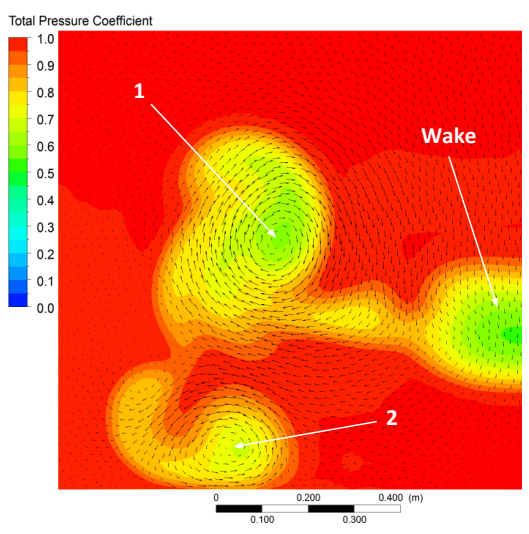

(b) $\mathrm{x} / \mathrm{L}=1.435$

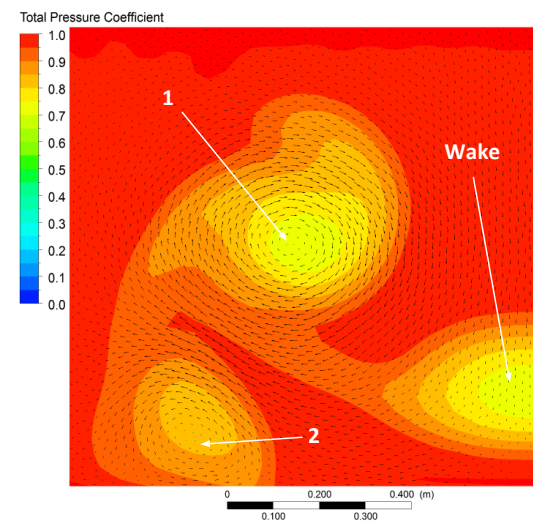

(c) $\mathrm{x} / \mathrm{L}=2.187$

Figure 11. Original Sunswift eVe

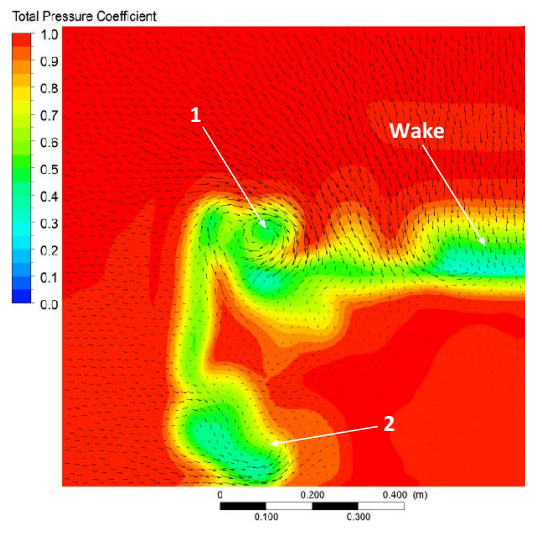

(a) $\mathrm{x} / \mathrm{L}=1.101$

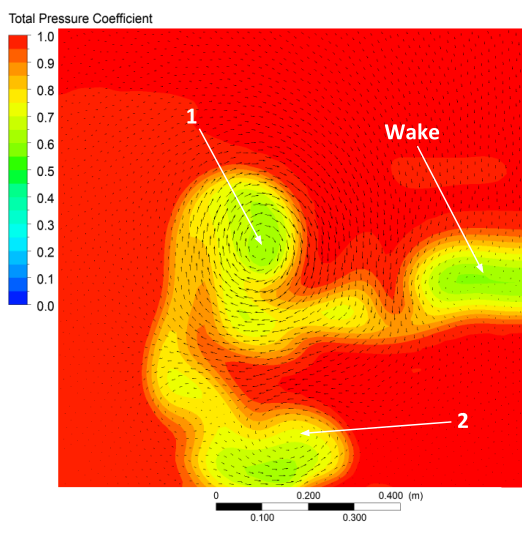

(b) $\mathrm{x} / \mathrm{L}=1.435$

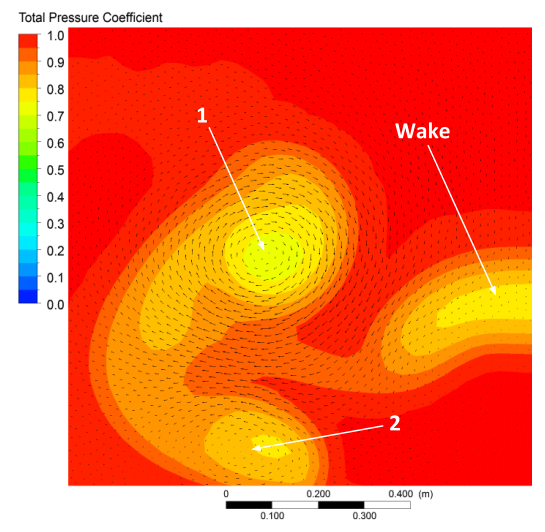

(c) $\mathrm{x} / \mathrm{L}=2.187$

Figure 12. Final trailing edge extension design

Both Figures 11 and 12 show the development of two trailing vortices; a primary vortex due to flow off the top rear wheel shoulder mixing with flow from the underside, and a smaller secondary vortex generated by the rear wheel. In comparison to Fig. 11, The vortex formation as a result of the trailing edge attachment, shown in Fig. 12, appears to be better defined. The lower pressure regions shown in Fig. 12 appear slightly smaller and the two vortices seem to have greater stability when compared with those in Fig. 11.

As mentioned earlier, the strong downwash observed off the rear of Sunswift eVe is evident in Fig. 11 and shows the downward motion of the low pressure wake. Conversely, Fig. 12 suggests that this effect has been practically eliminated through the boat-tailing design approach. This is made clear in Fig. 13, comparing the normalised x-velocity in the symmetry plane between the original car and trailing edge extension design. 


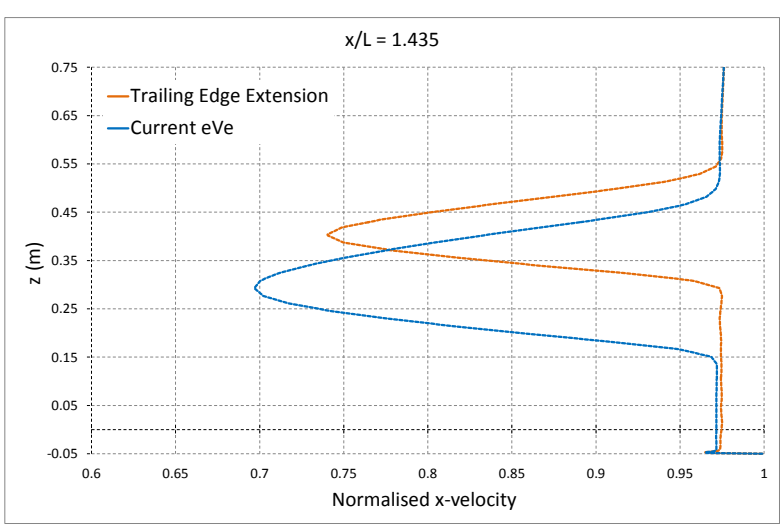

(a) $\mathrm{x} / \mathrm{L}=1.435$

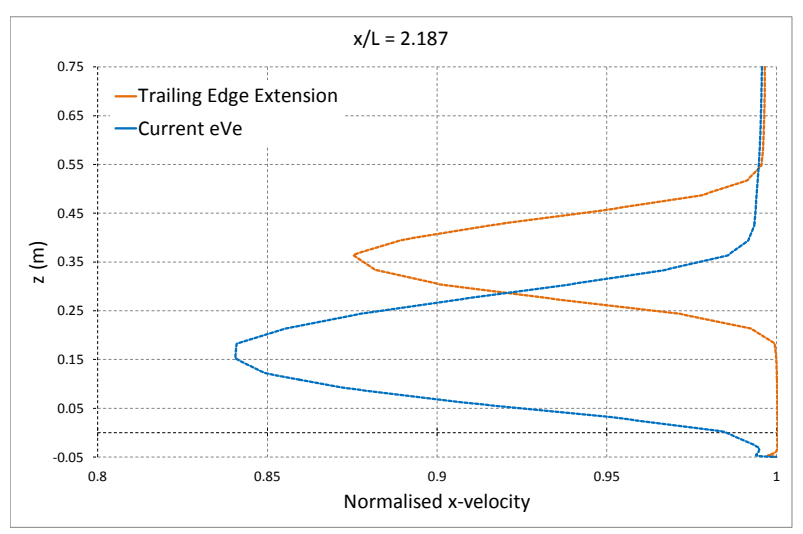

(b) $\mathrm{x} / \mathrm{L}=2.187$

Figure 13. Comparison of eVe with and without the trailing edge extension

Figure 13 shows that a narrower wake forms behind the trailing edge extension with less of a velocity deficit when compared with the original design of eVe. This wake is largely due to the attached boundary layer on the top and underside surfaces. Conversely, the wake generated by the current vehicle is larger due to separated flow off the top and underside surfaces. The difference between the plots in Fig. 13 clearly demonstrates the downward motion of the central low pressure wake behind Sunswift eVe, while the wake produced by the trailing edge extension maintains much more of a consistent level position. This reasonably level wake is also an indication of the strength of the two primary vortices forming behind the car. For the current vehicle design, the counter-rotation of these two primary vortices essentially funnels air downwards producing the observed downwash effect. By "filling in" the low pressure region behind the car with the trailing edge extension, the strength of the trailing vortices has been reduced, resulting in the mitigation of the downwash effect observed in the current vehicle.

\section{A. Land Speed Record Attempt}

Following on from the successful drag reduction predicted by the trailing edge extension, in preparation for the Sunswift team's world record attempt, a prototype was constructed. Medium density polystyrene foam was selected as the construction material for its favourable lightweight properties and proportional strength. The geometry of the trailing edge extension was decomposed into blocks, and each section was formed using a hot-wire cutter and then combined together to form the complete extension. Figure 14 displays the manufactured trailing edge extension used in the land speed record attempt.

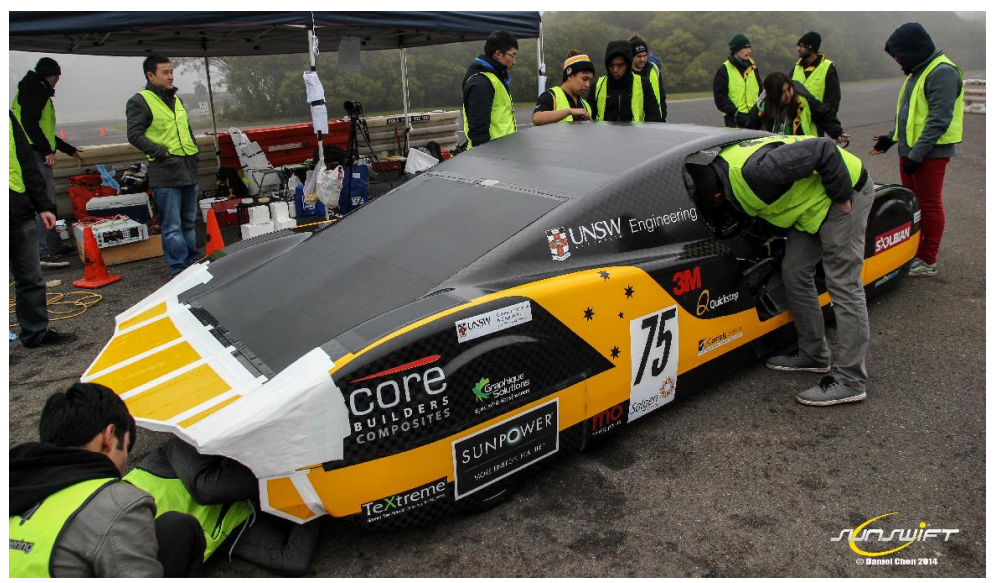

Figure 14. Final trailing edge design. Image credit Daniel Chen

In July 2014, the Sunswift team headed down to Melbourne to attempt to break the world record for the fastest long-range electric vehicle. During initial testing with the final trailing edge extension design, Sunswift eVe achieved a new personal best top speed of $132 \mathrm{~km} / \mathrm{h}$, with comments made by the driver believing that 
eVe could be pushed further. The previously documented top speed attained by eVe was $128 \mathrm{~km} / \mathrm{h}$ which was achieved during the 2013 WSC.

The dawn of the record attempt on the 23 July 2014 presented overcast weather with a heavy morning fog that gradually cleared throughout the day. As per the record requirements, eVe had to complete 119 laps of the $4.2 \mathrm{~km}$ circuit, covering this $500 \mathrm{~km}$ distance with an average speed greater than the previous record of $73 \mathrm{~km} / \mathrm{h}$.

The record attempt was successful, and the Sunswift team set an average speed of $107 \mathrm{~km} / \mathrm{h}$ over the $500 \mathrm{~km}$ distance, surpassing the previous record by $34 \mathrm{~km} / \mathrm{h}$.

\section{Conclusion}

$\mathrm{T}$ He study of the aerodynamic behaviour of eVe revealed a unique flow structure around the vehicle, displaying flow characteristics of both streamlined and bluff body automotive forms. The high presence of underbody suction was related to the production of undesirable flow effects, particularly the resulting incidence of yawed flow on the rear wheels, producing a relatively large unsteady wake that then forms a trailing vortex. Similarly, the turbulent interaction of flow off the top and underside rear surfaces is magnified by the formation of a low pressure region due to the truncated rear of the vehicle. Two primary counter-rotating vortices are seen to form as a result of this interaction.

This study focused on addressing the drag arising due to the low pressure wake forming behind the vehicle. A "boat-tailing" design strategy was implemented, and the resulting trailing edge extension design was predicted to reduce the drag of the vehicle by $10 \%$.

Design for low drag is beginning to play a greater role in the future of sustainable automotive transport. The challenges will forever remain in blending vehicle functionality and practicality with an optimised aerodynamic package. However, recent developments in alternative propulsion technologies present an opportunity for fundamental changes in vehicle architecture which could be exploited for low drag. ${ }^{5}$ As the external styling of a vehicle is a major selling point to the consumer market, radically designed low drag vehicle shapes are unlikely to catch on. Instead, aerodynamic optimisations of existing vehicle designs will likely achieve greater success, and will rely on the close integration of styling and aerodynamics departments within the automotive industry. ${ }^{5}$

\section{References}

${ }^{1}$ ANSYS, Inc., ANSYS Academic Research, Release 14.5, Help System, Meshing User's Guide.

${ }^{2}$ S. Diasinos, The Aerodynamic Interaction of a Rotating Wheel and a Downforce Producing Wing in Ground Effect, Phd Thesis, University of New South Wales, 2009.

${ }^{3}$ W. V. Duppen, I. P. Planquart, and J. Loeckx, Aerodynamic design of a low drag vehicle using the interaction between Computational Fluid Dynamics and wind tunnel tests, 2010

${ }^{4}$ R. H. Barnard, Road Vehicle Aerodynamic Design - An Introduction, Mechaero Publishing, 2 ed., 2001.

${ }^{5}$ G. Le Good, C. Johnson, B. Clough, and R. Lewis, The Aesthetics of Low Drag Vehicles, SAE International Journal of Engines, vol. 4, pp. 26382658, June 2011.

${ }^{6}$ A. Boulgakov, Sunswift IV Strategy for the 2011 World Solar Challenge, Honours Thesis, UNSW Australia, 2012.

${ }^{7}$ R. Palin, V. Johnston, S. Johnson, A. D'Hooge, B. Duncan, and J. I. Gargoloff, The Aerodynamic Development of the Tesla Model S - Part 1: Overview, Tech. Rep., SAE Technical Paper, April 2012.

${ }^{8}$ W. H. Hucho and G. Sovran, Aerodynamics of Road Vehicles, Annual Review of Fluid Mechanics, vol. 25, no. 1, pp. 485537, 1993.

${ }^{9}$ G. Tamai, The Leading Edge: Aerodynamic Design of Ultra-streamlined Land Vehicles, Robert Bentley, 1999. 